\title{
Control Board Digital Interface Input Devices - Touchscreen, Trackpad, or Mouse?
}

\section{International Symposium on Resilient Cognitive Systems - 2015 Resilience Week (RSW)}

Thomas A. Ulrich

Ronald L. Boring

Roger Lew

The INL is a

U.S. Department of Energy

National Laboratory

operated by

Battelle Energy Alliance

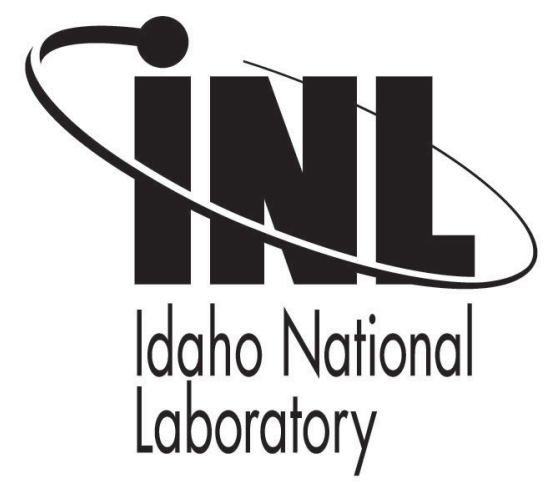

This is a preprint of a paper intended for publication in a journal or proceedings. Since changes may be made before publication, this preprint should not be cited or reproduced without permission of the author. This document was prepared as an account of work sponsored by an agency of the United States Government. Neither the United States Government nor any agency thereof, or any of their employees, makes any warranty, expressed or implied, or assumes any legal liability or responsibility for any third party's use, or the results of such use, of any information, apparatus, product or process disclosed in this report, or represents that its use by such third party would not infringe privately owned rights. The views expressed in this paper are not necessarily those of the United States Government or the sponsoring agency. 


\section{Control Board Digital Interface Input Devices - Touchscreen, Trackpad, or Mouse?}

\author{
Thomas A. Ulrich, \& Ronald L. Boring \\ Human Factors, Statistics, and Controls Department \\ Idaho National Laboratory \\ Idaho Falls, Idaho, 83415, USA \\ \{thomas.ulrich|ronald.boring\}@inl.gov
}

\author{
Roger Lew \\ Virtual Technology and Design Department \\ University of Idaho \\ Moscow, ID, 83843, USA \\ rogerlew@vandals.uidaho.edu
}

\begin{abstract}
The authors collaborated with a power utility to evaluate input devices for use in the human system interface (HSI) for a new digital Turbine Control System (TCS) at a nuclear power plant (NPP) undergoing a TCS upgrade. A standalone dynamic software simulation of the new digital TCS and a mobile kiosk were developed to conduct an input device study to evaluate operator preference and input device effectiveness. The TCS software presented the anticipated HSI for the TCS and mimicked (i.e., simulated) the turbine systems' responses to operator commands. Twenty-four licensed operators from the two nuclear power units participated in the study. Three input devices were tested: a trackpad, mouse, and touchscreen. The subjective feedback from the survey indicates the operators preferred the touchscreen interface. The operators subjectively rated the touchscreen as the fastest and most comfortable input device given the range of tasks they performed during the study, but also noted a lack of accuracy for selecting small targets. The empirical data suggest the mouse input device provides the most consistent performance for screen navigation and manipulating on-screen controls. The trackpad input device was both empirically and subjectively found to be the least effective and least desired input device.
\end{abstract}

Keywords-Human System Interface; Turbine Control System; Input Device; Touchscreen; Mouse; Trackpad; Nuclear Power Plant; Control Room Modernization

\section{INTRODUCTION}

As part of the United States Department of Energy Light Water Reactor Sustainability Program, Idaho National Laboratory (INL) has collaborated with a power utility to perform a range of usability, ergonomic, and human factors evaluations of the turbine control system (TCS) upgrade work at one of its nuclear power plants (NPPs), including the installation of the digital TCS. This cooperative research and development (R\&D) activity involves human factors experts, engineers, and licensed and formerly licensed nuclear power plant operators. The overall goal of the work is to evaluate the new TCS interface during early stages of development and provide guidance to the power utility on how to design the TCS so that it facilitates the safe and efficient operation of this NPP.

One step in the upgrade process involves evaluating the input device(s) required to control the digital TCS. The modifications to the control board resulting from the TCS upgrade needs to be designed so that it is easy for operators to use, satisfies Human Factors Engineering (HFE) criteria and guidelines (e.g., NUREG-0700), and supports the objective of providing acceptable human performance [1]. Achievement of these objectives includes evaluating qualified and available input devices. INL was therefore tasked to create a standalone TCS and a mobile hardware setup system that would allow them to conduct an input study at the NPP to evaluate operator preference and device effectiveness for trackpad, mouse, and touchscreen input devices that could be used with the TCS.

Careful consideration went into selecting three input devices for evaluation. The input devices would be used to control the cursor position and make selections within the new TCS. A keyboard was envisioned to provide operators with the ability to enter numerical data after the cursor is positioned in the appropriate entry field and the field has been activated via the mouse, trackpad, or touchscreen input device. The mouse and trackpad were considered because of the wide prevalence and subsequent user acceptance of these devices within the U.S. workforce [2]. The mouse, and associated keyboard, still continue to dominate as the most prevalent input device found within the workplace. The ubiquity of the mouse has allowed a large number of individuals to both accept the mouse as an input device and create a level of expertise in using the device. Both of these attributes make the mouse a strong candidate for serving as the input device for the new TCS. Trackpads are less prevalent, but still quite common within the workplace. Trackpads are commonly found on portable computers in which toting a mouse becomes cumbersome. The vast majority of portable laptop computers ship with a built in trackpad, which is evidence of the prevalence and acceptance of the trackpad device within the workplace

Touchscreens have become increasingly prevalent over the last decade. In particular touchscreens have emerged in two primary applications, which are smartphones and kiosks. Touchscreens have become the dominant input device for selfservice kiosks such as airline flight and baggage check-in, bank automated teller machines, and self-service checkout stands. A touchscreen serves as the input device within the display screen, which eliminates any peripheral hardware such as a keyboard or mouse. Additionally, there is little training required for an individual to effectively interact with the touchscreen input since the touch gesture interactions map intuitively to the natural way individuals interact with the 
physical world [3]. These touchscreen advantages likely account for the increasing prevalence of touchscreen devices, e.g., the large number of laptops featuring touchscreens since the advent of Windows 8 with its touch-centric human system interface (HSI).

Users do not necessarily prefer the quickest or most accurate input method and additional factors should be considered for selecting an appropriate input device. Indeed, [4] found that the touchscreen input for a single point selection with targets of sufficient size was faster than a mouse as an input device. However, participants' preference does not appear to be directly linked to optimal performance as evidenced by participants preferring the mouse even though it was noticeably slower than the touchscreen. Perhaps the accuracy of the mouse can account for the user preference for a suboptimal input device. Another experiment comparing touch input versus mouse manipulation found that the error rates for the mouse manipulation were significantly lower than those for the touch input, which may result in a more positive user preference for the mouse [5]. Presumably, the trackpad selection speed would reside somewhere between the mouse and touchscreen since it adopts the touch contact method from the touchscreen and retains the increased gain control from the mouse. Ultimately, the new TCS display can be designed to accommodate any of these three input devices to optimize the speed and accuracy of the interactions. However user preference becomes an increasingly important selection criterion because user acceptance and ergonomic comfort may become the biggest differences between these input devices. To investigate user preference and assess any ergonomic issues with the input devices this study allowed users to interact with the different devices to determine which they preferred and assess any difference in speed or accuracy.

\section{METHOD}

\section{A. Participants}

A total of 25 participants were recruited to take part in the input study. Licensed operators were the primary target population; however, a number of other plant personnel with turbine control experience were also recruited to participate in the study. Participating personnel included licensed reactor operators, senior reactor operators, instructors, and simulator support staff. Due to time limitations, only 8 participants were able to complete all aspects of the experiment. However, all participants that participated in the experiment were provided the opportunity to observe and interact with the prototype interface and each input device to ensure that their subjective reports using the different input devices for each of the interaction types captured in the survey possess validity

\section{B. Experimental Kiosk}

While the NPP has a fully functional training simulator, it must faithfully reflect the layout and functionality of the actual main control room. It is therefore not possible to modify its configuration to accommodate the anticipated installation of a new digital TCS. Thus, in order to conduct this input device study, INL had to 1) develop a functional prototype of the digital TCS, and 2) construct a kiosk that matched the shape and size of the control boards in the plant's main control room.
As such, a dynamic, functional prototype of the TCS HSI that interfaced with the plant simulator was developed as a purpose built Windows Presentation Foundation (WPF) application utilizing Western Services Corporation's (WSC) DLL interface library for .NET to communicate with the plant simulator [6]. That is, communication was enabled through a dynamic link library interface provided by the simulator vendor. The purpose of the prototype was to allow operators to operate the turbine system through the new digital control system, well before it was feasible to install the actual HSI and control system in the plant simulator, and without necessarily replicating every minute detail of the remaining control room interface. The TCS HSI was based on the prototype TCS HSI developed for a closely related NPP with modifications to allow the prototype to support administering experimental trials and recording the performance data. Additionally, the prototype was modified to ensure that it supported the interaction styles required to examine the effectiveness of each input device. The developed TCS HSI included similar features as would be included in the design for the NPP; however, some discrepancies existed since it was based off of a digital TCS developed separately for another NPP. The operators were made aware of the discrepancies to mitigate any potential confusion.

The power utility also provided INL with engineering drawings of the NPP control room and control boards to use as the basis for the construction of the kiosk. Figure 1 below shows the INL constructed kiosk matching the dimensions of the control board provided in the $\mathrm{EC}$, but is also modified slightly to make it easier to transport.
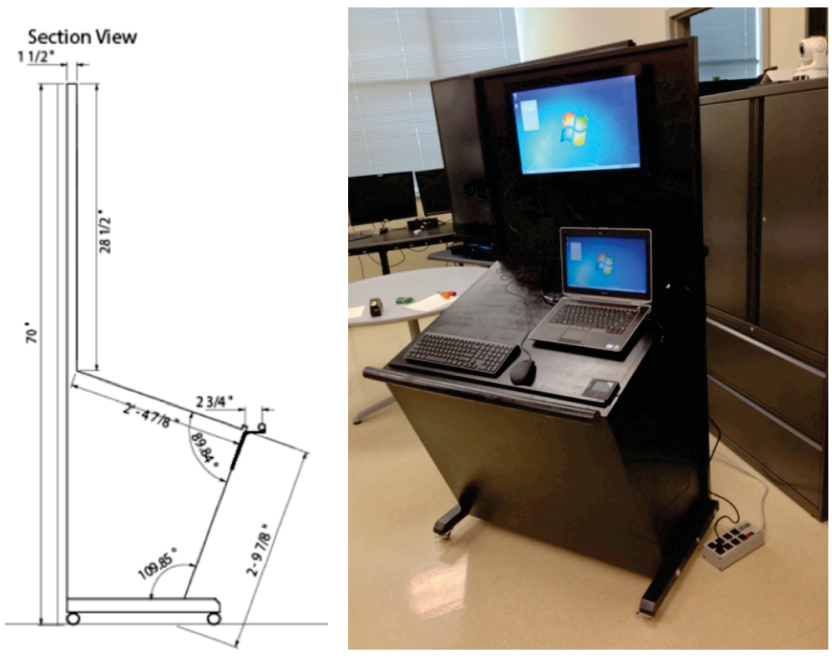

Figure 1. Engineering drawings and the fabricated kiosk supporting the TCS HSI and the proposed input device placements.

\section{Procedure}

Eight operators completed the full compliment of trials, including quantitative and qualitative measures, while 17 
participants familiarized themselves with the interaction methods and input devices but only completed the subjective questionnaire, i.e., qualitative measures. On the questionnaire, operators were first asked to rate the different input devices in terms of perceived difficulty for each of the following four types of interactions carried out with the TCS prototype:

- Large labeled navigation buttons selection

- Small arrow navigation buttons selection

- Text entry fields selection and numerical keyboard input

- Dropdown values selection was:

Operators were then asked to report the input device that

- Most preferred

- $\quad$ Least preferred

- Most accurate

- Least accurate

- Quickest

- Slowest

- Most comfortable

- Least comfortable

Operators were then asked to provide open-ended feedback concerning any difficulties reaching any of the devices, improvements to the inputs, and any additional inputs worth consideration for the new digital TCS.

In addition to collecting operator feedback via survey questions, the dynamic prototype of the digital TCS was programmed to collect objective performance data by measuring how much time it took operators to perform the various tasks. Specifically, the following objective metrics were collected:

- Time to complete trials

- Time to find target screen with arrow navigation

- Time to select the main menu button

- Time to navigate to a random screen

- Time to perform text entry fields selection and numerical input (i.e., Enter target load speed using the numeric keypad)

- Time to perform dropdown values selection (i.e., enter a target ramp rate using a cursor controlled dropdown box). This is also referred to as the selection entry control task.

\section{RESUlts}

\section{A. Quantitative Performance}

The small sample size of operators participating in the quantitative portion of the study resulted in low statistical power to resolve significant performance differences. Despite this limitation, we were able to identify a few significant differences in operator performance between the input devices.
2) Time to Complete Trials: Trial durations over the four trial sets were $\log _{10}$ transformed and aggregated for each participant (see Fig. 2a). The log transform was necessary to normalize heterogeneity in the dataset. A single factor ANOVA revealed a significant effect $(F(2,7)=3.98, p=$ 0.032 ). Two-tailed pairwise comparisons suggest the effect is due to operators completing trials faster with the mouse compared to the trackpad ( 11.8 vs. 17.8 seconds; $t(7)=3.10, p$ $=0.015)$. No significant differences of standard deviation of the $\log _{10}$ transformed durations were observed.

3) Time to Find Target Screen With Arrow Navigation: Participants were instructed to use the navigation arrow buttons located along the top of the prototype TCS to navigate to a given screen. No significant differences were found between the three input devices (see Fig. 2b). This does not necessarily suggest that differences do not exist between the input device conditions, just that no patterns are statistically reliable.

4) Time to Click Main Menu Button: The second of four trial sets started on a random screen and required operators to navigate to a target screen through the main menu link. An analysis was conducted on the time it took operators to navigate to the main menu. An ANOVA found significant differences due to device $(F(2,7)=5.78, p=0.011)$. The effect was carried by faster navigation with the mouse $(t(7)=$ 4.64, $p=0.003$; see Fig. 2c). Operators were $35 \%$ faster with the mouse.

5) Time to Navigate to Random Screen: The total time to navigate to a random screen was also examined (see Fig. 2d). No significant differences were found. This could be due to operators being unfamiliar with the TCS

6) Numeric Keypad Entry Task: Operators were required to change the Impulse-Out target load using a combination of a cursor coupled to the input device and a keyboard. The results found a small effect of device $(F(2,7)=4.04, p=$ 0.033 ; see Fig. 2e). The t-test suggests a marginal improvement of $25 \%$ might exist with the touchscreen compared to the trackpad $(t(7)=2.36, p=0.051)$.

7) Selection Entry Control Task: Operators were required to change the Impulse-Out load ramp rate using a cursor controlled selection box. The results found an effect of device $(F(2,7)=9.35, p=0.001)$ carried by a $35 \%$ improvement with a mouse over a trackpad $(t(7)=8.15, p<0.001$; see Fig. $2 \mathrm{f})$. The mouse also provided a $38 \%$ faster performance over the touchscreen $(t(7)=2.42, p=0.046)$.

\section{B. Perception of Input Devices}

In general, the operators reported favorable impressions of the mouse and touchscreen as evidenced below and in Figure 3. The trackpad received the smallest amount of favorable ratings with only a single operator ranking the trackpad input device the highest on any of the debrief questions. Each type of operator rating is described below. 
a. Log10 Duration

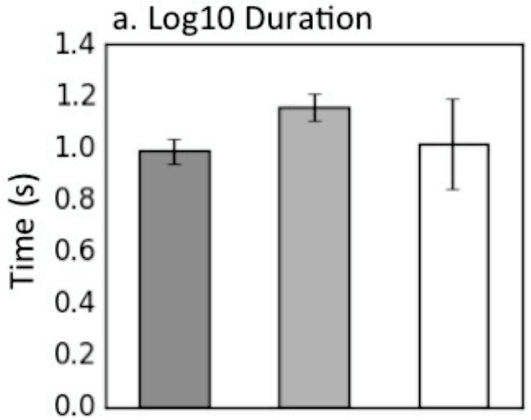

d. Navigate to Random

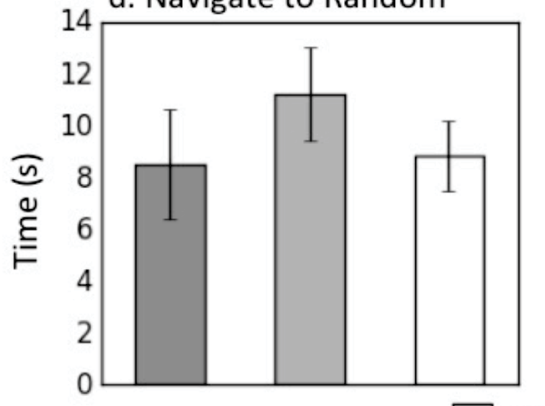

b. Navigate to Target

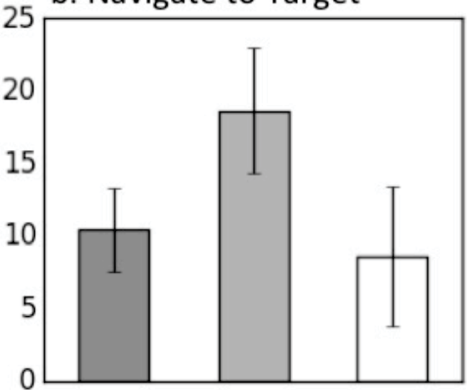

e. Numeric Keypad

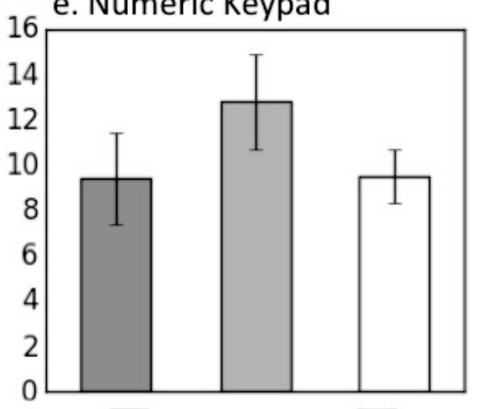

c. Navigate to Main
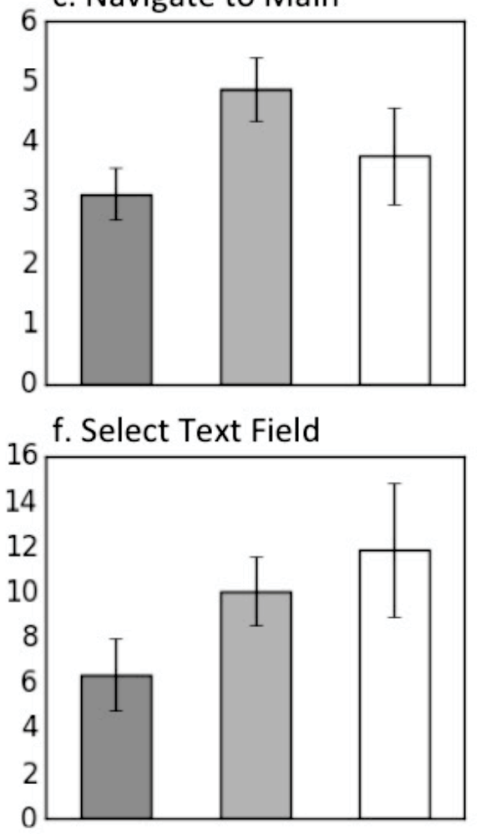

Touchscreen

Figure 2. Quantitative operator timing data for each input device. Panel a. depicts a Log10 transformation of trial completion times for each input device. Panel b. represents mean time to navigate to a target screen. Panel c. shows the mean time to navigate to the main navigation screen. Panel d. depicts the mean time to navigate to a random screen. Panel e. depicts the mean time to select a text field and complete the numeric keypad entry task. Panel f. shows the mean time to select a target text entry field. Error bars represent $90 \%$ confidence intervals.
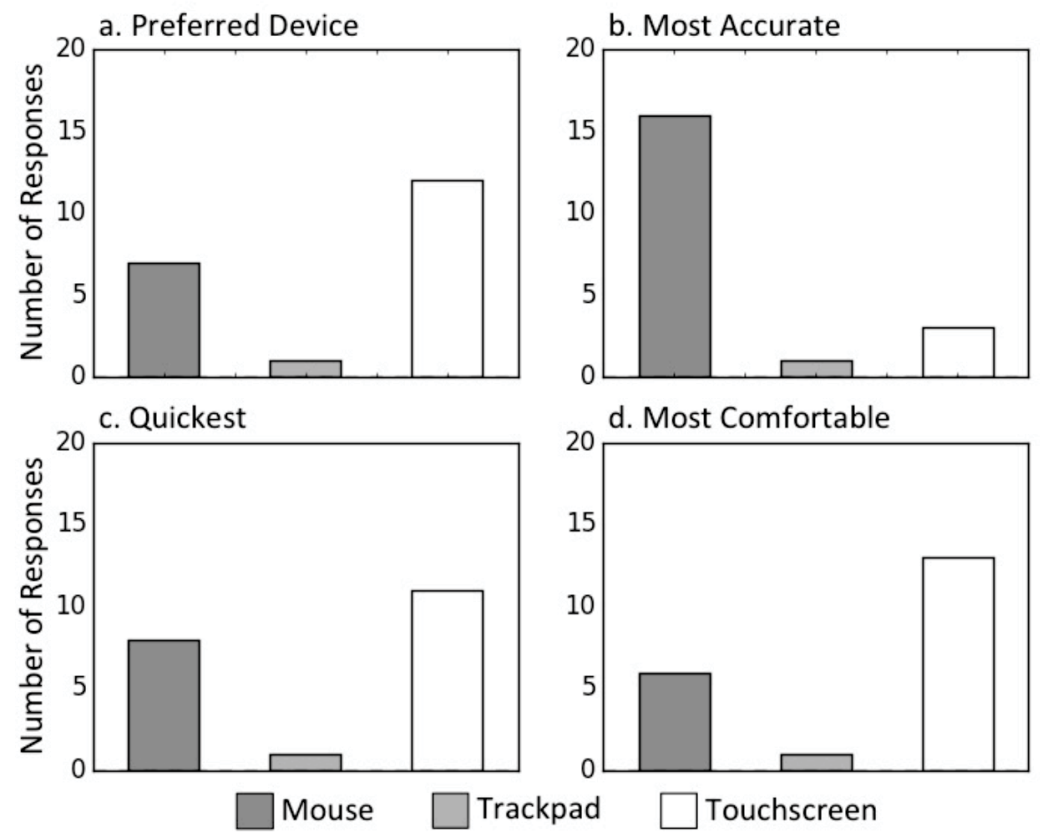

Figure 3. Histogram of operator perceived input device ratings. Panel a. depicts the frequency of operator reported preference for each input device. Panel b. represents the frequency of operator perceived highest accuracy device. Panel c. shows the frequency of operator perceived quickest input device. Panel d. depicts the operator perceived most comfortable input device. 
1) Ease of Use: Of the three input devices, the trackpad was rated as significantly more difficult than either the mouse or touchscreen to complete the tasks (data not shown). The mouse and the touchscreen were not significantly different in their difficulty ratings; however, the mouse was rated slightly less difficult to use than the touchscreen. Operators reported difficulties selecting small targets with the touchscreen, which likely resulted in the mouse and touchscreen difficulty rating differences. Larger targets would likely alleviate this difference in ratings, but there is an upper limit to how large the targets can be made, given the availability of screen space.

2) Preference: Many of operators reported preferring the touchscreen the most (see Fig. 3a). Conversely, many operators reported that the trackpad was their least preferred, while only one operator reporting it was their most preferred For the mouse, slightly more operators reported that it was their most preferred than those who reported it was their least preferred (seven vs. six respectively). This relatively contentious result for the mouse, in contrast to the very lopsided findings for touchscreen and trackpad is worth noting in the sense that it indicates potential disenfranchisement of a large minority of operators if the mouse is selected as the input device.

3) Accuracy: The majority of operators rated the touchscreen as the least accurate input device while three operators reported that it was the most accurate (see Fig. 3b). Only one operator reported the trackpad was the most accurate. Most of the operators rated the mouse as the most accurate.

4) Speed: A comparable number of operators reported the mouse and touchscreen as the fastest input device to complete the various tasks (see Fig. 3c). In contrast, only one operator reported the trackpad as the fastest input device.

5) Comfort: The majority of operators reported that the touchscreen was the most comfortable to operate (see Fig. 3d). In fact, no operator said that the touchscreen was the least comfortable. Additionally, the majority of operators reported that the trackpad was the least comfortable, with one operator saying it was the most comfortable. Operators' opinions were again most divided on the mouse, with eight operators reporting it was the least comfortable and six saying it was the most comfortable.

\section{Discussion}

The study produced a pattern of informative results to aid the new TCS design team's decision for an input device. The study revealed some statistically significant performance differences between the input devices. Notably, the trackpad was the lowest performing input device with the longest time to complete tasks and the most errors. Furthermore, the trackpad also received the lowest preference rating by the operators. As a result, the trackpad was not recommended for use as an input device for the TCS based on the results of this human factors study. The mouse and the touchscreen input devices both exhibited similar positive performance results, which makes them good candidates for selection as an input device for digital HSIs mounted on control boards. Based on the objective performance data, the touchscreen was the fastest of the input devices while the mouse was the most accurate. Interestingly, despite the lower accuracy as assessed by the object measures, the operators reported a preference for the touchscreen over the mouse input in general. This preference is likely driven by the ease of use afforded by touchscreen devices, such as smart phones, and their ubiquitous presence in society. However, as an input device for process control, this preference result should be carefully weighed against the accuracy tradeoff with the touchscreen. Process control relies on accurate manipulations of the interface to maintain the plant within safe operating parameters. As such, the accuracy of the mouse likely outweighs the preference for the touchscreen.

Though the performance differences observed in the data yielded statistically significant differences in terms of speed and accuracy, the amount of meaningful difference between the touchscreen and mouse input devices may prove nominal during actual daily use. The participants were unfamiliar with the interface, which likely impacted the captured timing data. The effect this lack of familiarity exhibited on the timing data can only be speculated on, but given more time using the interface, the timing and accuracy should improve. As a result the differences observed in this study may decrease. Fortunately, the accuracy was quite high and the amount of time required for any of the interaction tasks was small, which makes both the touchscreen and the mouse acceptable input devices. Overall, the mouse is the optimal input device based on these findings due to the slight accuracy advantage. Though the mouse was not the most preferred device, it still received positive subject ratings overall and would certainly be an acceptable choice for use with the new digital TCS HSI.

Additional considerations outside of performance and subjective ratings should be included in the decision for the input devices within NPP control rooms. A number of operators reported ergonomic issues concerning the touchscreen input device. Several operators reported they felt comfortable they could use the touchscreen given the reach envelope constrained by the length of the slanted lower control board section, but raised concerns that smaller statured individuals might experience difficulty. Due to this ergonomic issue, it is recommended that the touchscreen be avoided for applications with deep, slanted lower control board sections since a significant number of operators may not be able to easily reach the touch screen. Operators also reported using the mouse on the slanted lower control board section could over time become uncomfortable and cause strain on the wrist. The mouse is a good candidate, but if used it should be mounted on a folding tray so that it can be used comfortably on a flat horizontal surface to protect the wrist. The keyboard, which was intended to be included regardless of which input device was selected, also needs to be mounted and the folding tray could serve a dual purpose by also acting as the mount for the keyboard.

Beyond the ergonomic considerations, the physical constraints of the control boards and the available real-estate on the control boards must also be factored into the selection of the input device. The distance resulting from the size of the 
control board has already been mentioned in the section discussing ergonomic issues; however, the size of the touchscreen may also pose a problem. In order to make the touchscreen more usable, it would require larger target selection areas. These larger areas accommodate the size of the touch area on the finger to select a target. Larger target areas require more screen real estate, which increases the needed size of the touchscreen interface. Depending on the size constraints, the touch screen may not be a viable option. The mouse mounted on a folding tray can easily be fitted into the existing control board. As such, the mouse is was found to be the most desirable input device in terms of the physical constraints of the control board at least within the application investigated during this study.

The overall recommendation based on this study is to use the mouse input device mounted on a folding tray. The mouse input device mounted in this manner provides an ergonomic, accurate, and fast way for operators to interact with the new digit TCS HSI. The operators' favorable preference for the mouse also adds to its desirability as the input choice for application within NPP control boards.

\section{DISCLAIMER}

This work of authorship was prepared as an account of work sponsored by an agency of the United States Government. Neither the United States Government, nor any agency thereof, nor any of their employees makes any warranty, express or implied, or assumes any legal liability or responsibility for the accuracy, completeness, or usefulness of any information, apparatus, product, or process disclosed, or represents that its use would not infringe privately-owned rights. Idaho National Laboratory is a multi-program laboratory operated by Battelle Energy Alliance LLC, for the United States Department of Energy under Contract DEAC07-05ID14517.

\section{REFERENCES}

[1] J. M. O'Hara, et al., "Human-System Interface Design Review Guidelines," U.S. Nuclear Regulatory Committee, Washington, D.C., NUREG-0700, rev 2, 2002.

[2] V. Ahlstrom, and B. Kudrick, "Human Factors Criteria for the design and acquisition of non-keyboard interaction," U.S. Department of Transportation, Washington, D.C., 2004.

[3] C. Forlines, et al., "Touch vs. mouse input for tabletop displays," in Proceedings of Computer Human Interaction, New York, 2007, pp. 647656.

[4] A. Sears, and B. Shneiderman, (1991). High precision touchscreens: design strategies and comparisons with a mouse. Int. J. of Man-Machine Studies, pp. 593-613, vol 34(4), 1991.

[5] F. Sasangohar, et al., "Evaluation of mouse and touch input for a tabletop display using Fitts' reciprocal tapping task," Proceedings of the $53^{r d}$ Annual Meeting of the Human Factors and Ergonomics Society, Santa Monica, CA: 2009, pp. 8390843.

[6] R. Lew, et al., "A prototyping environment for research on humanmachine interfaces in process control: Use of microsoft WPF for microworld and distributed control system development, Proceedings of the International Symposium on Resilient Control Systems, 2014. 